\title{
DC System Modeling and AC/DC System Harmonic Calculation under Asymmetric Faults
}

\author{
Haifeng Li, Junlei Liu, Fengjiao Wang, Gang Wang \\ School of Electric Power, SCUT, Guangzhou, China \\ Email: lihf@scut.edu.cn, 1.junlei@scut.edu.cn \\ Received March, 2013
}

\begin{abstract}
The accurate DC system model is the key to fault analysis and harmonic calculation of AC/DC system. In this paper, a frequency domain analysis model of DC system is established, and based on it a unified fundamental frequency and harmonic iterative calculation method is proposed. The DC system model is derived considering the dynamic switching characteristic of converter and the steady-state response features of dc control system synchronously. And the proposed harmonic calculation method fully considers the AC/DC harmonic interaction and fault interaction under AC asymmetric fault condition. The method is used to the harmonic analysis and calculation of CIGRE HVDC system. Compared with those obtained by simulation using PSCAD/EMTDC software, the results show that the proposed model and method are accurate and effective, and provides the analysis basis of harmonic suppression, filter configuration and protection analysis in $\mathrm{AC} / \mathrm{DC}$ system.
\end{abstract}

Keywords: DC System Model; Harmonic Calculation; Switching Function; Asymmetric Faults

\section{Introduction}

Due to the significant advantages for long-distance, large-capability and cross-regional power transmission, DC transmission is widely used in China. In recent years, the pace of DC transmission construction is accelerating and several HVDC projects have been put into operation [1]. As is expected, by the time of 2020, there would have been 50 HVDC projects, and some of them are UHVDC transmission projects [2,3]. Along with a sound momentum in state grid construction, China will certainly become the one with the most complex AC/DC hybrid (HVDC and UHVDC included) and super large scale power grid in the world. As for the system, theoretical study and operating experience are inadequate at home and abroad. The harmonics problem is one of the problems in $\mathrm{AC} / \mathrm{DC}$ hybrid systems, which urgently needs to solve. However, harmonic interaction is extremely complex in $\mathrm{AC} / \mathrm{DC}$ hybrid system, due to the nonlinear characteristics of converter, so it is more difficult to conduct harmonic calculation [4]. And the key to solve the problem is to establish DC system model considering the interaction of $\mathrm{AC} / \mathrm{DC}$ system.

\footnotetext{
*The work is supported by National Natural Science Foundation of China (No.51007024, No.50977032), the Crucial Field and Key Breakthrough Project in "Guangdong-Hongkong" (No. 2009A0913 00011) and Guangdong Special Fund Project of Industry, University and Research Institute Collaboration(2011A090200074).
}

At present, harmonic analysis methods of HVDC system can be classified into two categories: time domain method and frequency domain method. The time domain method presents the converter on-off state by employing differential equation, and then harmonic calculation can be carried out by solving a group of differential equations in one period. Although the method is accurate, it cannot theoretically interpret the mechanism of harmonic generation, transformation and interaction. The frequency domain method mainly includes harmonic power flow method, iterative harmonic algorithm, and modulation harmonic analysis. The first two methods are accurate, but they cannot explain the mechanism of harmonic generation [4]. The frequency domain method based on modulation theory is widely used because it has advantages on revealing mechanism of harmonic generation, transformation and interaction [5-9]. In [5], switching function model of converter in normal steady state is established. The model use switching function to represent identical on-off and commutation process of converter valve. However, when an asymmetric fault occurs in the ac system, the unbalanced ac supply would make the converter operating under unbalanced conditions, during which the commutation overlaps and the switching instants of valves are unbalanced [9]. Then if the simple switching functions which are assumed to be of identical shape are used, unacceptable errors would be result in. Under unbalanced ac supply, the switching functions 
that include the effect of the unequal commutation overlaps are derived in [6], and that the varying firing angles are considered in [7]. But, neither of them considers the unbalanced commutation overlaps and the unbalanced switching instants of valves simultaneously. In [8], unified disturbing quantity is used to represent unbalance of firing angle of each valve. However, under unbalanced ac supply, different valve has different firing angle and the disturbing quantities are different. An advanced switching function model for the HVDC converter is described in [9]. In this model, both the effect of the varying switching instants and the unbalanced commutation overlaps in asymmetric situation are considered. In various AC/DC operating conditions, once the model is determined, harmonic transfer relationship between AC/DC systems will be clearly known. But the above papers so far do not mention how to obtain the needed parameters for switching function of converter according to the $\mathrm{AC} / \mathrm{DC}$ system operating conditions. It is the determination of these parameters that express interaction of $\mathrm{AC} / \mathrm{DC}$ system, which is also the key to applying modulation theory to harmonic calculation in AC/DC system.

The paper describes a DC system model that can be used in various operating condition in AC/DC system. This model considers the dynamic switching characteristics of converter and steady state response characteristics of DC control system simultaneously. Then combining the model with the topology of ac network, it also presents a harmonic calculation method that is suitable for ac asymmetric fault operating condition. The methods not only reveal the mechanism of fault interaction of the AC/DC system, but also that of harmonic interaction.

\section{Mathematics Model for Interaction of AC/DC System}

The operation state of ac/dc interconnected system is codetermined by AC system and DC system. In [10], (1) is used to express mathematics model for interaction of AC/DC system:

$$
\left\{\begin{array}{l}
f_{d c}\left(i_{1}, u_{1}\right)=0 \\
f_{a c}\left(i_{1}, u_{1}\right)=0
\end{array}\right.
$$

where, $u_{1}$ is fundamental frequency voltage of converter bus; $i_{1}$ is fundamental frequency current injected into ac system from dc system. Without considering interaction, $f_{d c}$ represents the relationships $u_{1}$ and $i_{1}$ determined by dc system, while $f_{a c}$ represents the relationships $u_{1}$ and $i_{1}$ determined by AC system.

Equation (1) only describes the relationship between fundamental frequency components. In fact, because converter is nonlinear, when the converter operates asymmetrically, the AC current injected by DC system consists of fundamental frequency components and harmonic components. Then, mathematics model of ac/dc interconnected system can be expressed as follows:

$$
\left\{\begin{array}{l}
\mathrm{F}_{d c}(\dot{\mathbf{I}}, \dot{\mathbf{U}})=0 \\
\mathrm{~F}_{a c}(\dot{\mathbf{I}}, \dot{\mathbf{U}})=0
\end{array}\right.
$$

where, $\dot{\mathbf{I}}=\left[\dot{I}_{1}, \dot{I}_{2}, \ldots \dot{I}_{l}\right], \quad \dot{\mathbf{U}}=\left[\dot{U}_{1}, \dot{U}_{2}, \ldots \dot{U}_{l}\right]$.

Under different operating conditions in ac/dc system, corresponding harmonics distribution can be obtained by solving equation (2). In that equation, $\mathrm{F}_{a c}$ decided by the ac system is linear and it can be easily acquired by ac network topology and parameters. Moreover, different frequency components are decoupling. While, $\mathrm{F}_{d c}$ decided by dc system is not only nonlinear, but also coupling between different frequency components. So it is very complicated. Therefore, under different operating conditions, the key to solving harmonics distribution of ac/dc system is accurately to derive corresponding $\mathrm{F}_{d c}$, that is to establish accurate mathematical model of DC system.

DC system contains converter, DC control system and DC transmission line. The model of converter will be firstly established.

\subsection{Advanced Switching Function Mode of Converter}

An advanced switching function model for the HVDC converter has been analyzed in [10]. In this model, the effects of unequal interval conduction of converter, the unbalanced commutation overlaps and non-linear commutating current are take into account in unbalanced ac supply. The model can be expressed as follows:

$$
\begin{gathered}
\dot{U}_{d(k)}=\sum_{m} \dot{S}_{u(k-m)} T_{1} \dot{U}_{(m)}^{( \pm)}=\sum_{m}\left(\dot{S}_{u(k-m)}^{+} \dot{U}_{(m)}^{+}+\dot{S}_{u(k-m)}^{-} \dot{U}_{(m)}^{-}\right) \\
\dot{I}_{(l)}^{( \pm)}=T_{2} \sum_{n} \dot{S}_{i(l-n)} \dot{I}_{d(n)}=\sum_{n} \dot{S}_{i(l-n)}^{( \pm)} \dot{I}_{d(n)}
\end{gathered}
$$

where, $\dot{S}_{u(k)}, \dot{S}_{i(k)}$ are $k$ th vector of three-phase voltage and current of switch function;

$$
\begin{aligned}
\dot{U}_{(m)}^{( \pm)} & =\left[\begin{array}{ll}
\left\langle u_{i}^{+}\right\rangle_{m} & \left\langle u_{i}^{-}\right\rangle_{m}
\end{array}\right]^{T}, \dot{I}_{(l)}^{( \pm)}=\left[\begin{array}{ll}
\left\langle i_{i}^{+}\right\rangle_{l} & \left\langle i_{i}^{-}\right\rangle_{l}
\end{array}\right]^{T} ; \\
T_{1} & =\left[\begin{array}{ll}
1 & 1 \\
a^{2} & a \\
a & a^{2}
\end{array}\right], T_{2}=\frac{1}{3}\left[\begin{array}{ccc}
1 & a & a^{2} \\
1 & a^{2} & a
\end{array}\right], a=e^{j 2 \pi / 3} .
\end{aligned}
$$

Based on [10], on condition that $\dot{U}_{1}, I_{d 0}, \alpha_{0}$ is known, the advanced switch function $S$ can be achieved, as following:

$$
S=\left[\dot{S}_{u}, \dot{S}_{i}\right]=\mathrm{G}\left(\dot{U}_{1}, I_{d 0}, \alpha_{0}\right)
$$

where, $\dot{U}_{1}$ is fundamental frequency voltage of converter bus, $I_{d 0}$ is dc current on dc side, $\alpha_{0}$ is firing instruction angle. 


\subsection{Nodal Impedance Matrix of DC Network}

Equivalent impedance of dc network consists of three parts, which are harmonic impedance of dc lines, of $\mathrm{dc}$ filters and smoothing reactor, and of the converter on offside. With DC network topology and parameters, the equivalent harmonic impedance $\left(Z_{d(n)}\right)$ on DC side is calculated in [11], and then the relationship between harmonic current and voltage can be described as follows:

$$
\dot{I}_{d(n)}=\dot{U}_{d(n)} / Z_{d(n)} \quad(n \neq 0)
$$

where, $Z_{d(n)}$ is deduced by assumed that offside convertor is symmetric operating when ac system occurs asymmetric fault on rectifier or inverter side. That is, the equation only fits for the situation that the converter on the fault side is the only harmonic source. In order to achieve consistent expression in modeling DC system, equivalent DC impedance of converter on dc side can be defined as:

$$
Z_{d 0}=U_{d 0} / I_{d 0}
$$

where, $U_{d 0}, I_{d 0}$ are respectively DC components of DC side voltage and current. With (3), (6) and (7), DC components of the current on DC side can be obtained, as well as each harmonic component:

$$
\dot{I}_{d(n)}=\frac{1}{Z_{d(n)}} \sum_{m}\left(\dot{S}_{u(n-m)}^{+} \dot{U}_{m}^{+}+\dot{S}_{u(n-m)}^{-} \dot{U}_{m}^{-}\right)
$$

Substitute (8) into (4), fundamental frequency and each harmonic components of $\mathrm{AC}$ current injected by converter can be expressed as:

$$
\begin{gathered}
\dot{I}_{(l)}^{+}=\sum_{n} \frac{\dot{S}_{i(l-n)}^{+}}{Z_{d(n)}} \sum_{m}\left(\dot{S}_{u(n-m)}^{+} \dot{U}_{m}^{+}+\dot{S}_{u(n-m)}^{-} \dot{U}_{m}^{-}\right) \\
\dot{I}_{(l)}^{-}=\sum_{n} \frac{\dot{S}_{i(l-n)}^{-}}{Z_{d(n)}} \sum_{m}\left(\dot{S}_{u(n-m)}^{+} \dot{U}_{m}^{+}+\dot{S}_{u(n-m)}^{-} \dot{U}_{m}^{-}\right)
\end{gathered}
$$

Based on (9) and (10), (11) can be formed:

$$
\left[\begin{array}{c}
\dot{I}_{1}^{+} \\
\dot{I}_{1}^{-} \\
\dot{I}_{2}^{+} \\
\dot{I}_{2}^{-} \\
\vdots \\
\vdots \\
\dot{I}_{l}^{+} \\
\dot{I}_{l}^{-}
\end{array}\right]=\left[\begin{array}{cccccccc}
A_{11}^{+} & A_{11}^{-} & A_{12}^{+} & A_{12}^{-} & \cdots & \cdots & A_{1 m}^{+} & A_{1 m}^{-} \\
B_{11}^{+} & B_{11}^{-} & B_{12}^{+} & B_{12}^{-} & \cdots & \cdots & B_{1 m}^{+} & B_{1 m}^{-} \\
A_{21}^{+} & A_{21}^{-} & A_{22}^{+} & A_{22}^{-} & \cdots & \cdots & A_{2 m}^{+} & A_{2 m}^{-} \\
B_{21}^{+} & B_{21}^{-} & B_{22}^{+} & B_{22}^{-} & \cdots & \cdots & B_{2 m}^{+} & B_{2 m}^{-} \\
\vdots & \vdots & \vdots & \vdots & \ddots & & \vdots & \vdots \\
\vdots & \vdots & \vdots & \vdots & & \ddots & \vdots & \vdots \\
A_{l 1}^{+} & A_{l 1}^{-} & A_{l 2}^{+} & A_{l 2}^{-} & \cdots & \cdots & A_{l m}^{+} & A_{l m}^{-} \\
B_{l 1}^{+} & B_{l 1}^{-} & B_{l 2}^{+} & B_{l 2}^{-} & \cdots & \cdots & B_{l m}^{+} & B_{l m}^{-}
\end{array}\right]\left[\begin{array}{c}
\dot{U}_{1}^{+} \\
\dot{U}_{1}^{-} \\
\dot{U}_{2}^{+} \\
\dot{U}_{2}^{-} \\
\vdots \\
\vdots \\
\dot{U}_{m}^{+} \\
\dot{U}_{m}^{-}
\end{array}\right]
$$

where, $\quad A_{l m}^{+}=\sum_{n} \frac{\dot{S}_{i(l-n)}^{+} \dot{S}_{u(n-m)}^{+}}{Z_{d(n)}} ; A_{l m}^{-}=\sum_{n} \frac{\dot{S}_{i(l-n)}^{+} \dot{S}_{u(n-m)}^{-}}{Z_{d(n)}}$

$$
B_{l m}^{+}=\sum_{n} \frac{\dot{S}_{i(l-n)}^{-} \dot{S}_{u(n-m)}^{+}}{Z_{d(n)}} ; B_{l k}^{-}=\sum_{n} \frac{\dot{S}_{i(l-n)}^{-} \dot{S}_{u(n-m)}^{-}}{Z_{d(n)}} .
$$

The relation matrix of (11) is marked as $\mathbf{Y}$. According to the above derivation, $\mathbf{Y}$ is the function of $I_{d 0}$, $\alpha_{0}, U_{d 0}$ and $U_{d 0}$, which can be expressed as:

$$
\mathbf{Y}=\mathrm{H}\left(\dot{U}_{1}, I_{d 0}, \alpha_{o}, U_{d 0}\right)
$$

Then (11) can be expressed as:

$$
\mathrm{J}\left(\dot{\mathbf{U}}, \dot{\mathbf{I}}, I_{d 0}, \alpha_{0}, U_{d 0}\right)=0
$$

Comparing (13) with (2), when $I_{d 0}, \alpha_{f}, U_{d 0}$ are known, based on (13), DC system model $\mathrm{F}_{d c}$ will be acquired. Therefore, how to obtain $I_{d 0}, \alpha_{0}, U_{d 0}$ that change with operating conditions plays the key role in building DC equivalent model. In (13), $\alpha_{0}$ rest with trigger control system, and $\left(I_{d 0}, U_{d 0}\right)$ is operating point of dc system. All these are determined by control system and $\mathrm{AC} / \mathrm{DC}$ operating state.

\section{DC System Model Considering Interaction of AC/DC System}

Fast and various-way modulation is one of the main advantages of dc system. According to operating conditions of ac/dc system and pre-determined control strategies, dc system will automatically modulate. Different dc systems use different control methods, even in the same dc system, the control strategies are varied with different operating conditions. In this paper, take standard testing system of CIGRE HVDC as an example to describe the modeling process of steady state response of control system.

Steady state $U_{d}-I_{d}$ characteristic curve of CIGRE HVDC is shown in Figure 1. $U_{d}$ and $I_{d}$ are respectively dc voltage and current of converter export, while practical calculation, they are respectively substituted by $U_{d 0}$, $I_{d 0}$. The fine line shows steady-state operation characteristic of the inverter controller when the rectifier operates at the min trigger angle $\left(\alpha_{\min }\right)$ control. And the heavy line describes steady-state operation characteristic of the rectifier controller when the inverter operates at constant extinction angle $\left(\gamma_{0}\right)$ control.

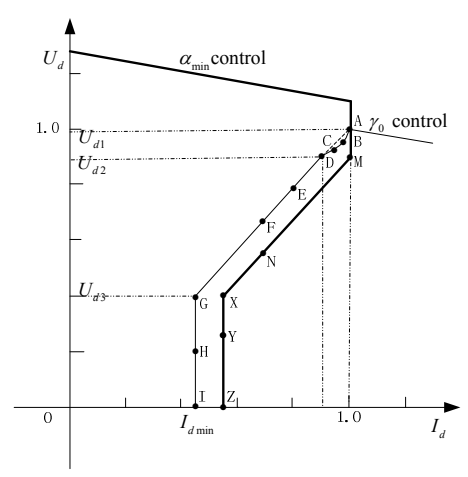

Figure 1. Steady state $U_{d}-I_{d}$ curve of CIGRE HVDC. 


\subsection{Steady-state Response Model of Rectifier Control System}

As shown in Figure 1, in different operating conditions, the relation of $U_{d}$ and $I_{d}$ can be expressed with a piecewise function. For the convenience of calculation, the control region of current error control (CEC), as shown with the pitch arc ACD, adopts linear fitting, as shown with the dashed line AD. So, according to Figure 1, the relationship of $U_{d}$ and $I_{d}$ can be express as:

$$
I_{d}=\left\{\begin{array}{l}
1 ; U_{d 1}<U_{d} \leq 1 \\
k_{1} U_{d}+b_{1} ; U_{d 2}<U_{d} \leq U_{d 1} \\
k_{2} U_{d}+b_{2} ; U_{d 3}<U_{d} \leq U_{d 2} \\
I_{d \text { min }} ; 0<U_{d} \leq U_{d 3}
\end{array}\right.
$$

where, $U_{d 1}, U_{d 2}, U_{d 3}, k_{1}, k_{2}, b_{1}, b_{2}, I_{d \min }$ are parameters of control system, and $U_{d 0}$ can be achieved by the following equation:

$$
U_{d 0}=\sum_{m}\left(\dot{S}_{u(n-m)}^{+} \dot{U}_{m}^{+}+\dot{S}_{u(n-m)}^{-} \dot{U}_{m}^{-}\right)
$$

By substitution (15) into (14), the relationship between $I_{d 0}$ and $\dot{U}$ can be found. From (15), the model considers the effects of harmonic components of ac side on the dc system operating points.

In general, when fault occurs in ac system of rectifier side, $\left(I_{d 0}, U_{d 0}\right)$ are determined by (14) and (15), while, the firing instruction angle of rectifier can be derived by the min trigger angle control.

$$
\alpha_{0}=\alpha_{\text {min }}
$$

\subsection{Steady Response Model of Inverter Control System}

From Figure 1, the relationship between $U_{d 0}$ and $I_{d 0}$ at the inverter export also can be found; such as (14), only the control parameters are different from rectifier. Under ac fault condition, $\alpha_{0}$ is known on the rectifier side. While, the inverter side known is extinction angle $\gamma_{0}$. The control diagram of constant extinction angle is shown in Figure 2.

When the system operates in normal static condition, steady state model of inverter is similar to that of rectifier. Then $\gamma_{0}$ can replace $\alpha_{0}$ directly. However, when ac system occurs asymmetric fault, $\alpha_{0}$ cannot be directly replaced by $\gamma_{0}$ any more. But the $\alpha_{0}$ is needed in modeling the advanced switching function model. So, the

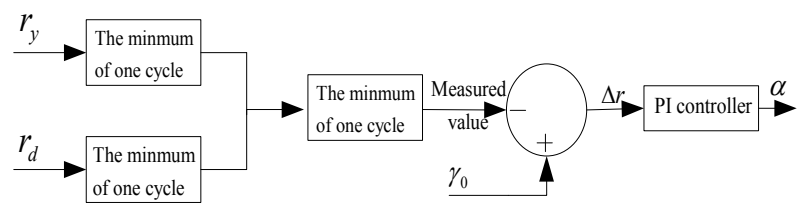

Figure 2. The control diagram of $\gamma_{0}$. relationship between $\gamma_{0}$ and $\alpha_{0}$ should be established in the inverter side. The formulas of $\gamma_{m n}, \alpha_{m n}, \mu_{m n}$ $(m, n=a, b, c)$ in inverter can be found from:

$$
\begin{aligned}
& \gamma_{m n}+\alpha_{m n}+\mu_{m n}=\pi \\
& \mu_{m n}=\cos ^{-1}\left(\cos \alpha_{m n}-2 X_{r} I_{d} / U_{m n}\right)-\alpha_{m n} \\
& \alpha_{m n}= \begin{cases}0 & \Delta \varphi_{m n}>\alpha_{o} \\
\alpha_{o}-\Delta \varphi_{m n} & \Delta \varphi_{m n} \leq \alpha_{o}\end{cases}
\end{aligned}
$$

where, $\Delta \varphi_{m n}$ is phase separation between mn commutating voltage and corresponding trigger reference phasor [9] .

Simultaneously, with equations (17) and (18), (20) can be established:

$$
\cos \alpha_{m n}=\frac{2 X_{T} I_{d 0}}{U_{1 m n}}-\cos \gamma_{m n}
$$

Based on constant extinction control theory, it is certain that there should be extinction angle of at least one pair of valves that equals $\gamma_{0}$, but which pair is unknown. Hereby, method of trial is adopted to derive $\gamma_{0}$ from $\alpha_{0}$. The process: Supposed $\gamma_{c a}, \gamma_{a b}$ and $\gamma_{b c}$ are equal to $\gamma_{0}$, then (18) and (19) are respectively plugged into corresponding $U_{m n}$ and $\Delta \varphi_{m n}$, three trigger instruction angles $\alpha_{0 c a}, \alpha_{0 a b}, \alpha_{0 b c}$ can be achieved. It is certain that $\alpha_{0}$ is one of the three. Assuming that $\alpha_{0}=\alpha_{0 c a}$, then with (16), (17) and (18), practical extinction angles of each converter valves $\gamma_{c a}, \gamma_{a b}, \gamma_{b c}$ will be known. If the one is less than $\gamma_{0}$, and then $\alpha_{0 c a}$ can be ruled out. If there is at least one that equals $\gamma_{0}$, and the rest are all greater than $\gamma_{0}$, then $\alpha_{0 c a}$ is firing instruction angle. Similar methods can be employed to verify $\alpha_{0 a b}, \alpha_{0 b c}$. Finally, the real firing instruction angle $\alpha_{0}$ can be obtained. That is, for inverter, according to $\gamma_{0}, \alpha_{0}$ can be given by:

$$
\alpha_{0}=C_{1}\left(\dot{U}_{1}, I_{d 0}\right)
$$

\subsection{System Model Considering AC/DC System Interaction}

With control method of CIGRE HVDC system, the relationship between $U_{d 0}$ and $I_{d 0}$ has been given above. In fact, for a DC system, no matter what control strategies are adopted, based on steady state control curve of control system, the relation of $U_{d 0}$ and $I_{d 0}$ can be expressed as:

$$
I_{d 0}=C_{2}\left(U_{d 0}\right)
$$

Above all, it is known that whether rectifier or inverter, based on steady state response of control system, corresponding $I_{d 0}, \alpha_{0}$ and $U_{d 0}$ can be acquired. And then, the dc model will be established, which considers ac/dc interaction. 


\section{AC/DC System Fault Calculation and Harmonic Calculation}

When an asymmetric fault occurs in the ac system, converter still operates symmetrically because the AC supply is still symmetrical. The simple case is not included in the paper. This paper mainly studies ac/dc system fault and harmonics calculation when ac system occurs asymmetrical fault. In the previous part, DC system model that considers the effect of dc control system has been analyzed. That is, $\mathrm{F}_{d c}$ is obtained and called as DC constraint condition of $\mathrm{AC} / \mathrm{DC}$ fault and harmonics calculation. Correspondingly, $\mathrm{F}_{a c}$ is called as $\mathrm{AC}$ constraint condition of AC/DC fault and harmonics calculation. Under ac fault condition, on the basis of ac network topology structure and boundary conditions of asymmetric fault, $F_{a c}$ can be obtained. Take single phase grounding fault of converter bus as an example to illustrate that process of modeling $\mathrm{F}_{a c}$. Figure 3 shows the network structure of ac/dc interconnected power grid.

Because the ac system is linear, the fundamental frequency and each harmonic component are independent of each other in the ac system. Steps for modeling fundamental frequency impedance matrix are as follow:

1) Positive sequence network of fundamental frequency

Where, $F$ is the fault position. Positive sequence equivalent voltage and impedance will be easily acquired by using superposition theorem:

$$
\begin{gathered}
\dot{U}_{f 0}^{+}=Z_{\Sigma 1}^{+} \dot{I}_{1}^{+}+\frac{Z_{c 1}^{+}}{Z_{s 1}^{+}+Z_{c 1}^{+}} E_{s} \\
Z_{\Sigma 1}^{+}=Z_{s 1}^{+} \| Z_{c 1}^{+}
\end{gathered}
$$

2) negative sequence network of fundamental frequency

According to Figuer 5, negative sequence equivalent voltage and impedance can be expressed as:

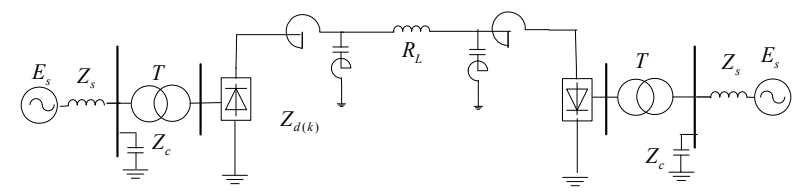

Figure 3. AC/DC transmission system.

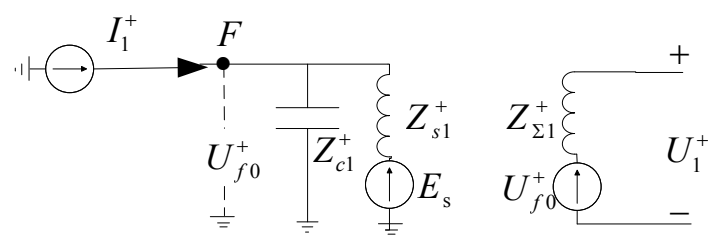

Figure 4. Positive sequence network.

$$
\begin{aligned}
\dot{U}_{f 0}^{-} & =Z_{\Sigma 1}^{-} \dot{I}_{1}^{-} \\
Z_{\Sigma 1}^{-} & =Z_{\Sigma 1}^{+}
\end{aligned}
$$

3) zero sequence network of fundamental frequency

For converter station of 12-pulse, in order to reduce harmonics, transformer $\mathrm{Y} / \mathrm{Y}$ is equipped at the high voltage terminal, and transformer $\mathrm{Y} / \Delta$ at the low voltage terminal. Generally, the neutral point is not grounded on valve side of transformer $\mathrm{Y} / \mathrm{Y}$, so there is no zero-sequence current through this kind of transformer. But for transformer $\mathrm{Y} / \Delta$, the neutral point in star coil is directly grounded, thus for this kind of transformer, zero sequence network is closed on the side of star coil. So, zero-sequence impedance of $Y / \Delta$ transformer should be contained in zero-sequence network of ac/dc system. Zero sequence equivalent impedance can be archived by Figure 6:

$$
Z_{\Sigma 1}^{0}=Z_{c 1}^{0}\left\|Z_{s 1}^{0}\right\| Z_{T \Delta 1}^{\prime 0}
$$

Based on the sequence boundary conditions of singlephase grounding, compound sequence network of single-phase grounding can be established. Then fundamental frequency impedance matrix can be obtained, and the voltage and current of the fundamental frequency $Z_{1}$ satisfy:

$$
\left[\begin{array}{c}
\dot{U}_{1}^{+} \\
\dot{U}_{1}^{-}
\end{array}\right]=\left[\begin{array}{lll}
Z_{111} & Z_{112} & Z_{113} \\
Z_{121} & Z_{122} & Z_{123}
\end{array}\right]\left[\begin{array}{c}
\dot{I}_{1}^{+} \\
\dot{I}_{1}^{-} \\
E_{s}
\end{array}\right]=Z_{1}\left[\begin{array}{c}
\dot{I}_{1}^{+} \\
\dot{I}_{1}^{-} \\
E_{s}
\end{array}\right]
$$

where,

$$
\begin{aligned}
& Z_{111}=Z_{122}=\left(1+Z_{\Sigma 1}^{+} / Z_{\Sigma 1}\right) Z_{\Sigma 1}^{+} ; \\
& Z_{112}=Z_{121}=\left(Z_{\Sigma 1}^{+}\right)^{2} / Z_{\Sigma 1} ; Z_{123}=\left(Z_{\Sigma 1}^{+}\right)^{2} /\left(Z_{s 1}^{+} Z_{\Sigma 1}\right) ; \\
& Z_{113}=\left(1+Z_{\Sigma 1}^{+} / Z_{\Sigma 1}\right) Z_{\Sigma 1}^{+} / Z_{s 1}^{+} ; \quad Z_{\Sigma 1}=Z_{\Sigma 1}^{+}+Z_{\Sigma 1}^{-}+Z_{\Sigma 1}^{0} .
\end{aligned}
$$

Similarly to $Z_{1}$, harmonics impedance matrix of ac system can be achieved. Then, harmonic voltage and current satisfy:
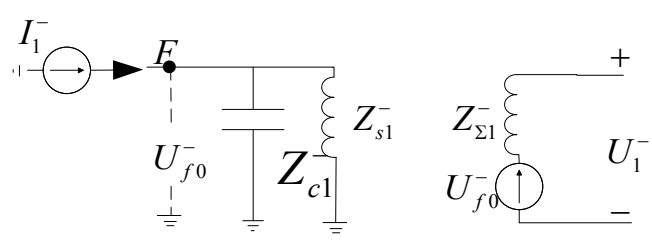

Figure 5. Negative sequence network.

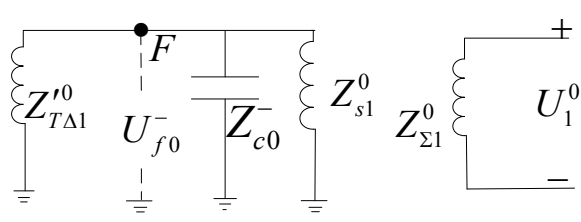

Figure 6. Zero sequence network. 


$$
\left[\begin{array}{c}
\dot{U}_{l}^{+} \\
\dot{U}_{l}^{-}
\end{array}\right]=\left[\begin{array}{cc}
Z_{l 11} & Z_{l 12} \\
Z_{l 21} & Z_{l 22}
\end{array}\right]\left[\begin{array}{c}
\dot{I}_{l}^{+} \\
\dot{I}_{l}^{-}
\end{array}\right]=Z_{l}\left[\begin{array}{c}
\dot{I}_{l}^{+} \\
\dot{I}_{l}^{-}
\end{array}\right]
$$

where, $Z_{l}$ is $l$ th harmonic impedance matrix. And then, $\mathrm{F}_{a c}$ can be established by (28) and (29). In different ac asymmetrical fault conditions, with $\mathrm{F}_{a c}$ and $\mathrm{F}_{d c}$, the corresponding results of $\mathrm{AC} / \mathrm{DC}$ fault and harmonics calculation can be turned out.

In fact, $\mathrm{F}_{a c}$ are linear, which can be easily obtained by topology of the AC/DC system. However, because of involving trigonometric function and piecewise function, $\mathrm{F}_{d c}$ are nonlinear and complicated. Therefore, the equation (2) cannot be solved directly, an iterative calculation method is proposed in this paper, which is described as follows: (the numbers in brackets denote iteration number, 0 represents initial value).

1) Initializing: calculate $Z_{d(n)}, Z_{l}, Z_{1}, \dot{S}_{u}^{ \pm}[0]$ and $\dot{S}_{i}^{ \pm}[0]$ in normal operation.

2) Figure out initial voltage of commutating bus. Without considering the change of dc system under fault situation, supposed $\dot{I}_{1}^{+}[0]=\dot{I}_{1 N}, \quad\left(\dot{I}_{1 N}\right.$ is fundamental frequency current injected into ac from dc in normal steady operation), $\left.\dot{I}_{1}^{-}[0] \overline{z_{1}}[0], \dot{I}_{l}^{ \pm}[0]\right]^{ \pm}[0]$, and these are put into (28) and (29), $U_{1}^{ \pm}[0]$ and $U_{l}[0]$ can be obtained.

3) Modify dc network matrix Y. $U_{d 0}[0]$ can be solved with $\dot{U}_{1}^{ \pm}[0], \quad \dot{S}_{u}^{ \pm}[0]$ and (15). Substitute $U_{d 0}[0]$ into (22), and $I_{d 0}[0]$ can be turned out. Then, $Z_{d 0}[1]$ can be achieved by using (7). According to $\dot{U}_{1}^{ \pm}[0]$, $I_{d 0}[0]$ and $\alpha_{\min }\left(\right.$ or $\left.\gamma_{0}\right), \alpha_{m n}[1], \mu_{m n}[1], \theta_{m n}[1]$ can be obtained, then improved converter switch function model $\dot{S}_{u}^{ \pm}[1], \dot{S}_{i}^{ \pm}[1]$ can be established. Finally, modified $\mathbf{Y}[1]$ can be got by using $\dot{S}_{u}^{ \pm}[1], \dot{S}_{i}^{ \pm}[1], Z_{d n}$ and $Z_{d 0}[1]$.

4) Modify the current injected into ac from dc (Fundamental frequency and each harmonic components included). $\dot{I}_{1}^{ \pm}[1]$ and $\dot{I}_{l}^{ \pm}[1]$ can be calculated with $\mathbf{Y}[1]$, $\dot{U}_{1}^{ \pm}[0]$ and (11).
5) Modify converter bus voltage. With $\dot{I}_{1}^{ \pm}[1], \dot{I}_{l}^{ \pm}[1]$, (28) and (29), $\dot{U}_{1}^{ \pm}[1]$ and $\dot{U}_{l}^{ \pm}[1]$ can be figured out through reconditions fault calculation.

Repeat from step (3) to (5) until the convergence condition is met. That is, the changes of voltage of converter bus are not noticeable.

$$
\max \left(\left|\dot{U}_{l}^{ \pm}[k+1]-\dot{U}_{l}^{ \pm}[k]\right|\right)<\varepsilon
$$

From the above steps, each re-conducted fault calculation not only modifies fundamental frequency voltage, but also harmonic voltage. Therefore, this means is called unified fundamental frequency and harmonic iterative calculation method. Base on step (3), the influence of ac on dc can be embodied by considering the effect of $\dot{U}_{1}$ on $\alpha_{m n}, \mu_{m n}$ and $\theta_{m n}$ into account. And base on step (4) and (5), the influence of ac on dc can be embodied by considering the effect of $S$ and $\left(I_{d 0}, U_{d 0}\right)$ on $\dot{\mathbf{I}}$. That is, the method fully considers the $\mathrm{AC} / \mathrm{DC}$ fault interaction under AC asymmetric fault condition.

\section{Simulation Verification}

Extensive simulation studies based on the CIGRE HVDC Benchmark model is done to test the proposed approach. Different conditions of asymmetric faults occur in ac system (without commutation failure) are taken into account, and the calculation results are compared with those obtained from simulation using PSCAD/EMTDC simulation software.

Constant current control, the minimum trigger angle control and voltage-dependent current order limit (VD$\mathrm{COL}$ ) are used at the rectifier. While, the inverter is equipped with constant extinction angle control, VDCOL control and current error control (CEC). The iterative convergence standard is $\varepsilon=1 \times 10^{-3}$, and after 4 times iterations, a part of results are shown in Table 1-2 where $I_{(k)}$ is the amplitude of $k$ th harmonic current; $R_{g}$ is the fault resistor.

\begin{tabular}{|c|c|c|c|c|c|c|c|c|c|}
\hline \multicolumn{5}{|c|}{ rectifier } & \multicolumn{5}{|c|}{ inverter } \\
\hline \multirow{2}{*}{$R_{g}$} & \multicolumn{2}{|c|}{$\dot{U}_{1}\left(\mathrm{kV} /{ }^{\circ}\right)$} & \multicolumn{2}{|c|}{$I_{d 0}(\mathrm{kA})$} & \multirow{2}{*}{$R_{\mathrm{g}}$} & \multicolumn{2}{|c|}{$\dot{U}_{1}\left(\mathrm{kV} /{ }^{\circ}\right)$} & \multicolumn{2}{|c|}{$\alpha_{0}(\operatorname{radian})$} \\
\hline & calculation & simulation & calculation & simulation & & calculation & simulation & calculation & simulation \\
\hline 0 & $\begin{array}{c}168.24 \angle-105.64 \\
112.03 \angle 69.06 \\
56.94 \angle 85.00\end{array}$ & $\begin{array}{c}169.27 \angle-106.16 \\
112.80 \angle 68.52 \\
57.91 \angle 84.25\end{array}$ & 1.341 & 1.405 & 70 & $\begin{array}{c}171.87 \angle 106.61 \\
16.38 \angle-15.66 \\
8.02 \angle 1.67\end{array}$ & $\begin{array}{c}169.09 \angle 106.54 \\
15.81 \angle-15.21 \\
7.87 \angle 1.59\end{array}$ & 2.332 & 2.319 \\
\hline 10 & $\begin{array}{c}180.22 \angle-114.02 \\
101.18 \angle 81.28 \\
51.17 \angle 98.97\end{array}$ & $\begin{array}{c}181.80 \angle-114.81 \\
101.83 \angle 80.56 \\
51.84 \angle 98.25\end{array}$ & 1.409 & 1.480 & 90 & $\begin{array}{c}175.86 \angle 107.82 \\
13.18 \angle-10.91 \\
6.47 \angle 4.53\end{array}$ & $\begin{array}{c}173.10 \angle 107.74 \\
12.72 \angle-12.45 \\
6.35 \angle 4.42\end{array}$ & 2.354 & 2.365 \\
\hline 50 & $\begin{array}{c}229.32 \angle-122.01 \\
56.62 \angle 106.75 \\
29.29 \angle 123.99\end{array}$ & $\begin{array}{c}234.42 \angle-121.97 \\
58.27 \angle 107.12 \\
29.96 \angle 123.92\end{array}$ & 1.825 & 1.821 & 110 & $\begin{array}{c}178.27 \angle 108.64 \\
11.00 \angle-9.70 \\
5.406 \angle 6.43\end{array}$ & $\begin{array}{c}175.54 \angle 108.56 \\
10.64 \angle-10.64 \\
5.33 \angle 6.30\end{array}$ & 2.376 & 2.386 \\
\hline 100 & $\begin{array}{c}258.05 \angle-120.29 \\
35.58<118.59 \\
18.01 \angle 135.23\end{array}$ & $\begin{array}{c}257.77 \angle-120.37 \\
35.51 \angle 118.64 \\
18.22 \angle 135.17\end{array}$ & 1.994 & 1.938 & 130 & $\begin{array}{c}179.67 \angle 109.23 \\
9.32 \angle-8.33 \\
4.64 \angle 7.81\end{array}$ & $\begin{array}{c}177.27 \angle 109.16 \\
9.10 \angle-9.36 \\
4.56 \angle 7.63\end{array}$ & 2.391 & 2.401 \\
\hline
\end{tabular}

Table 1. The fault calculation and simulation results of asymmetric faults occur at ac side. 
Table 2. The harmonic calculation and simulation results of asymmetric faults occur at ac side.

\begin{tabular}{cccccccccc}
\hline \multirow{2}{*}{$\begin{array}{c}\text { fault } \\
\text { side }\end{array}$} & $R_{\mathrm{g}}$ & \multicolumn{2}{c}{$\dot{I}_{3}(\mathrm{kA})$} & \multicolumn{2}{c}{$\dot{I}_{5}(\mathrm{kA})$} & \multicolumn{2}{c}{$\dot{U}_{d 2}(\mathrm{kV})$} & \multicolumn{2}{c}{$\dot{U}_{d 4}(\mathrm{kV})$} \\
\cline { 3 - 9 } & & calculation & simulation & calculation & simulation & calculation & simulation & calculation & simulation \\
\hline \multirow{6}{*}{ rectifier } & 0 & 0.469 & 0.487 & 0.224 & 0.215 & 234.348 & 225.376 & 20.235 & 25.704 \\
& 50 & 0.545 & 0.549 & 0.150 & 0.164 & 218.974 & 212.534 & 16.421 & 15.130 \\
& 10 & 0.321 & 0.317 & 0.077 & 0.074 & 128.389 & 119.215 & 8.172 & 7.626 \\
& 70 & 0.185 & 0.187 & 0.024 & 0.025 & 79.094 & 73.860 & 3.761 & 3.905 \\
\multirow{6}{*}{ inverter } & 0.137 & 0.149 & 0.012 & 0.013 & 46.798 & 46.809 & 1.801 & 1.939 \\
& 110 & 0.115 & 0.111 & 0.0084 & 0.0079 & 37.652 & 36.639 & 1.347 & 1.180 \\
& 130 & 0.0922 & 0.0956 & 0.0062 & 0.0057 & 31.848 & 30.730 & 1.006 & 1.100 \\
\hline
\end{tabular}

In the tables, the harmonic values are of the phase which has the highest amplitude of harmonic currents. From Tables 1-2, it's clear that, for $\dot{U}_{1}, I_{d 0}, \dot{I}_{3}$ and $\dot{U}_{d 2}$, the amplitude relative error between simulation value and calculation value is less than $5 \%$. While, for $\dot{I}_{5}$ and $\dot{U}_{d 4}$, the amplitude relative error between simulation value and calculation value is less than $10 \%$. And for the $\alpha_{0}$, the absolute error between simulation value and calculation value is less than 1 . The data analysis indicated that the proposed harmonic calculation method can provide precise and reliable results.

\section{Conclusions}

This paper describes a model of de transmission system that considers converter dynamic switch characteristics and steady-state response features of dc control system. Based on the model, a unified fundamental frequency and harmonic iterative calculation method is also proposed. This method is suitable for ac/dc fault and harmonic calculation when ac system occurs asymmetrical fault. The model established in this paper can make dc system have direct interface with ac system, no matter what operating conditions in $\mathrm{AC} / \mathrm{DC}$ system. And the methods not only reveal the mechanism of fault interaction of the AC/DC system, but also that of harmonic interaction.

In addition to providing a reliable model for DC system, the method provides quantitative analysis basis of harmonic mitigation, filter configuration and protection analysis in $\mathrm{AC} / \mathrm{DC}$ system.

\section{REFERENCES}

[1] Y.B. Shu, "Present Status and Prospect of HVDC Transmission in China," High Voltage Engineering, Vol. 30, No. 11, 2004, pp.1-2.

[2] Q. Y. Yuan, "Present Status and Prospect of Ultre-HVDC Transmission in China," Power System Technology, Vol.
29, No. 14, 2005, pp.1-3.

[3] H. T. Su, Q. Xu, Y. Wu, "Study on Market Demand of UHVDC Power Transmission in China," Power System Technology, Vol. 29, No. 2, 2005, pp.1-4.

[4] Y. Zeng, Z. Ren, T. Yu, "Study on Multi-Harmonic Sources in AC/DC Hybrid Transmission System Based on Modulation and Iterative Harmonic Analysis Method," Power System Technology, Vol. 30, No. 11, 2006, pp.27-31.

[5] Q. Q. Ru, L. W. Jiao, Y. Zheng, Y. X. Ni, S. S. Chen, F. 1. $\mathrm{Wu}$, "Modeling and Simulation of HVDC with Dynamic Phasor,"Proceedings of the CSEE, Vol. 23, No. 12, 2003, pp.28-32.

[6] L. H. Hu, R. E. Morrison. "The Use of Modulation Theory to Calculate the Harmonic Distortion in HVDC Systems Operating on An Unbalanced Supply," IEEE Transactions On Power Electronics, Vol. 12, No. 2,1997, pp.973-980. doi:10.1109/59.589796

[7] A. R. Wood, J. Arrillaga, "HVDC Convertor Waveform Distortion: A Frequency-domain Analysis," IEEE Proceedings Generations Transmissions Distibution, Vol. 1, No. 142, 1995, pp. 88-96.

[8] Y. L. Ma, X. N. Xiao, X. Jiang, “Analysis of the Impact of AC System Single-Phase Earth Fault on HVDC," Proceedings of the CSEE, Vol. 62, No. 11, 2006, pp.144-149.

[9] G. Wang, Z. K. Li, H. F. Li, "Dynamic Phasor Model of the Converter of the AC/DC System," Proceedings of the CSEE,2010, Vol. 30, No. 1, 2010, pp.59-64.

[10] H. F. Li, P. Zhang, G. Wang, G. L. Zhu, "Performance of Directional Protection Based on Variation of Power-frequency Components in HVDC/AC Interconnected System Part One DC-system Equivalent Impedance of Power-frequency Component Variation," Automation of Electric Power Systems, Vol. 33, No. 9, 2009, pp.41-46.

[11] Z. K. Li, G. Wang, H. F. Li, "An Analysis and Calculation Method of Harmonic Interaction between $\mathrm{AC}$ and DC System under Asymmetric Operating Conditions," Automation of Electric Power Systems, 2010, Vol. 34, No. 6, 2010, pp. 42-47. 